\title{
A new apparatus for electron tomography in the scanning electron microscope
}

Cite as: AIP Conference Proceedings 1667, 020013 (2015); https://doi.org/10.1063/1.4922569

Published Online: 25 June 2015

V. Morandi, M. Del Marro, P. Maccagnani, L. Masini, A. Migliori, L. Ortolani, G. Pallocca, A. Pezza, M. Rossi, G. Sberveglieri, M. Vittori-Antisari, P. Vinciguerra, and M. Ferroni

\section{ARTICLES YOU MAY BE INTERESTED IN}

Contrast and resolution versus specimen thickness in low energy scanning transmission electron microscopy

Journal of Applied Physics 101, 114917 (2007); https://doi.org/10.1063/1.2745333

Scanning electron microscopy of thinned specimens: From multilayers to biological samples Applied Physics Letters 90, 163113 (2007); https://doi.org/10.1063/1.2724917

Preface: Nanoforum 2014

AIP Conference Proceedings 1667, 010001 (2015); https://doi.org/10.1063/1.4922556

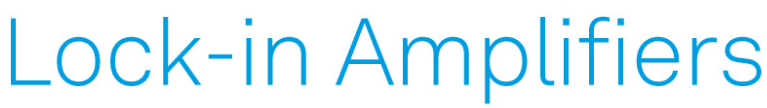
up to $600 \mathrm{MHz}$
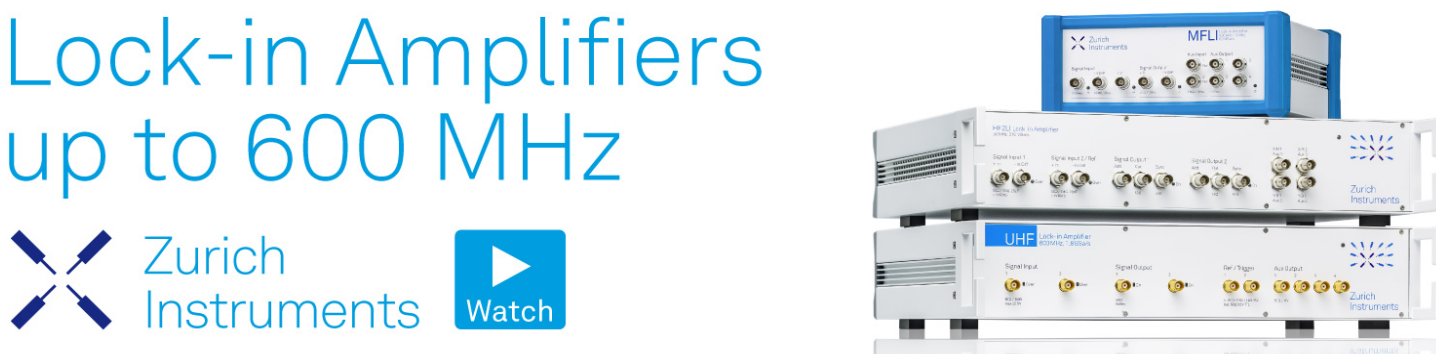


\title{
A new apparatus for electron tomography in the Scanning Electron Microscope
}

\author{
V. Morandi ${ }^{1, \text { a) }}$, M. Del Marro ${ }^{2}$, P. Maccagnani ${ }^{1}$, L. Masini $^{1}$, \\ A. Migliori ${ }^{1}$, L. Ortolani ${ }^{1}$, G. Pallocca ${ }^{2}$, A. Pezza ${ }^{1}$, M. Rossi $^{3}$, \\ G. Sberveglieri ${ }^{4}$, M. Vittori-Antisari ${ }^{5}$, P. Vinciguerra ${ }^{2}$, M. Ferroni ${ }^{3}$ \\ ${ }^{1}$ CNR-IMM Sezione di Bologna, via Gobetti 101, 40129 Bologna, Italy \\ ${ }^{2}$ ASSING S.P.A., via E. Amaldi 14, 00016 Monterotondo (Rome), Italy \\ ${ }^{3}$ Dip.to di Scienze di Base e Applicate per l'Ingegneria and Centro di Ricerca per le Nanotecnologie Applicate \\ all'Ingegneria (CNIS), Università degli Studi di Roma "Sapienza", Via A. Scarpa, 00161 Rome, Italy \\ ${ }^{4}$ SENSOR Lab, Dip.to di Ingegneria dell'Informazione, Università degli Studi di Brescia and CNR-INO, \\ Via Valotti 9, 25123 Brescia, Italy \\ ${ }^{5}$ Unità Tecnica Tecnologie dei Materiali, ENEA Centro Ricerche Casaccia, Via Anguillarese 301, \\ 00123 S. Maria di Galeria (Rome), Italy

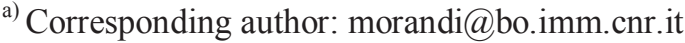

\begin{abstract}
The three-dimensional reconstruction of a microscopic specimen has been obtained by applying the tomographic algorithm to a set of images acquired in a Scanning Electron Microscope. This result was achieved starting from a series of projections obtained by stepwise rotating the sample under the beam raster. The Scanning Electron Microscope was operated in the scanning-transmission imaging mode, where the intensity of the transmitted electron beam is a monotonic function of the local mass-density and thickness of the specimen. The detection strategy has been implemented and tailored in order to maintain the projection requirement over the large tilt range, as required by the tomographic workflow. A Si-based electron detector and an eucentric-rotation specimen holder have been specifically developed for the purpose.
\end{abstract}

\section{INTRODUCTION}

In the field of three-dimensional (3D) material characterization, X-Ray-based Tomography and Electron Tomography (ET) are the leading techniques [1-3]. Both methodologies share the approach to retrieve a 3D density distribution map for the sample starting from a series of projection-images taken at different angles and reconstructing the spatial distribution using the backprojection algorithm underlying the principles of tomography [4]. In Figure 1, the volume analyzed, and the size of the discrete volume element of the tomogram (namely voxel), are graphically represented for the principal tomographic techniques. These two figures of merit represent the capability of tomography to explore biological or inorganic structures at different length scale and resolution. X-Ray Tomography is undoubtedly the most important one for industrial applications and provides the reconstruction of milli- to micro- metric objects. On the other hand ET, which is primarily carried out in the Transmission Electron Microscope (TEM), takes advantage from its magnifying capability and achieves nanometric resolution in the 3D reconstruction of small volumes. Indeed, the best resolution, as small as $1 \mathrm{~nm}$, has been pursued for volumes below one cubic micrometer [5]. This makes ET of great interest in many different research fields, from biology to materials science, disciplines where electron microscopy is widely used and appreciated.

The Scanning Electron Microscope (SEM) is conventionally operated for the visualization of the surface of bulk specimens, but recent implementations allowed one to explore the inner structure of samples [6]. Presently, the 3D methods in SEM have to be destructive as the specimen volume is serially sectioned by focused ion beam or 
mechanical cutting. The 3D reconstruction of relatively large volumes is thus obtained by reassembling the images obtained through this slice-and view approach.

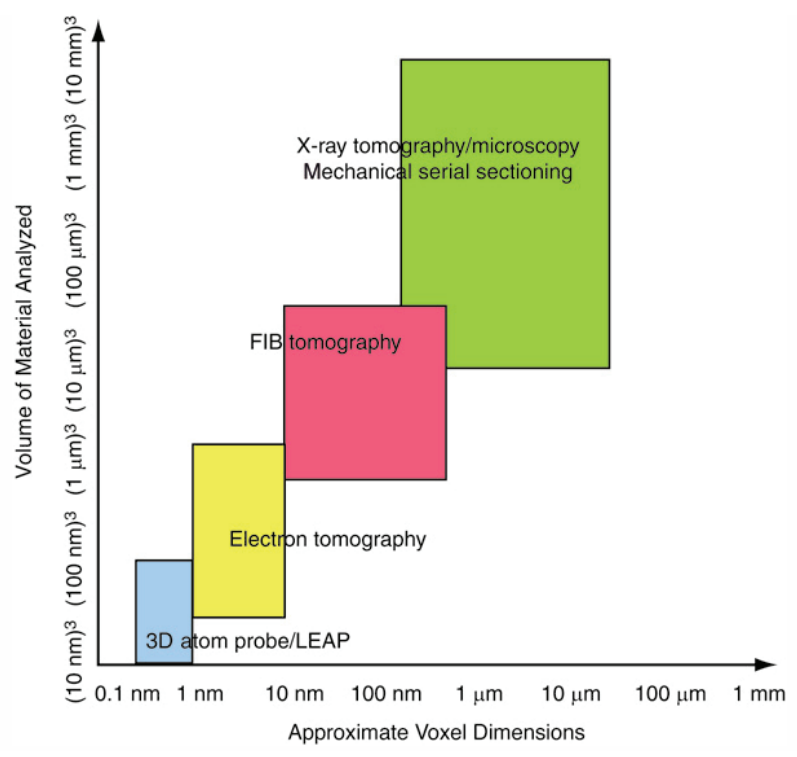

FIGURE 1: Voxel size and volume analyzed for the principal tomographic techniques. Reproduced with permission from Ref. 3. Copyright (C) Materials Research Society.

In this paper we will highlight the implementation of an innovative electron tomography system for the SEM operated in scanning-transmission imaging mode, composed by a completely dedicated detection system, namely electron detector, sample holder and analogue/digital signal processing system. The achievements of this implementation of tomography and the potential and the capabilities of this novel 3D imaging technique are summarized and discussed for its practical application.

\section{EXPERIMENTAL SET-UP}

The implementation of electron tomography in the SEM - a ZEISS LEO 1530 equipped with a Schottky emitter is based on the signal provided by the Scanning-Transmission (STEM) imaging mode. The STEM imaging mode is already implemented in modern SEMs, where specific detector and holder for the thin specimen are available from all the principal manufacturers. For a specimen sufficiently thin to provide electron transparency, the intensity of the incoherently scattered electrons is a monotonic function of local sample thickness and mass-density [6]. In such a condition, a STEM image represents a useful projection of the structure and thus a set of STEM images acquired at different tilt, corresponding to different directions for the projection, could be used for a tomographic reconstruction. Typically, a series of about 100 images covering the tilt range between $-60^{\circ}$ and $+60^{\circ}$ is required. The principal constraint for the success of tomography using scanning-transmission imaging is to maintain the monotonic variation of the mass-thickness contrast over the whole tilt range. However, for a practical implementation of electron tomography in the SEM, a dedicated adjustable STEM detector and a precise specimen rotation system are also required.

\section{STEM detector}

STEM imaging in the SEM takes advantage from some peculiar characteristics of the experimental set-up: the size of the focused electron beam ultimately defines the nanometric resolution, the absence of post-specimen lenses allows one to obtain images free from chromatic aberration; the relatively large distance between the sample and the microscope column facilitates the operation of the rotation holder. The use of STEM for tomography requires an acquisition system capable of collecting the transmitted electron over a large and adaptable angular collection range. 
Therefore, the optimization of design and performance for the detector is required in order to comply with local variations of composition or projected thickness for the specimen.

Beam acceleration voltage and specimen-detector distance are the basic operative parameters to govern the detection angles that becomes particularly crucial in case of relatively large specimen thickness, because the possibility to govern the collection angles is essential to maintain the proper image contrast when the specimen is rotated.
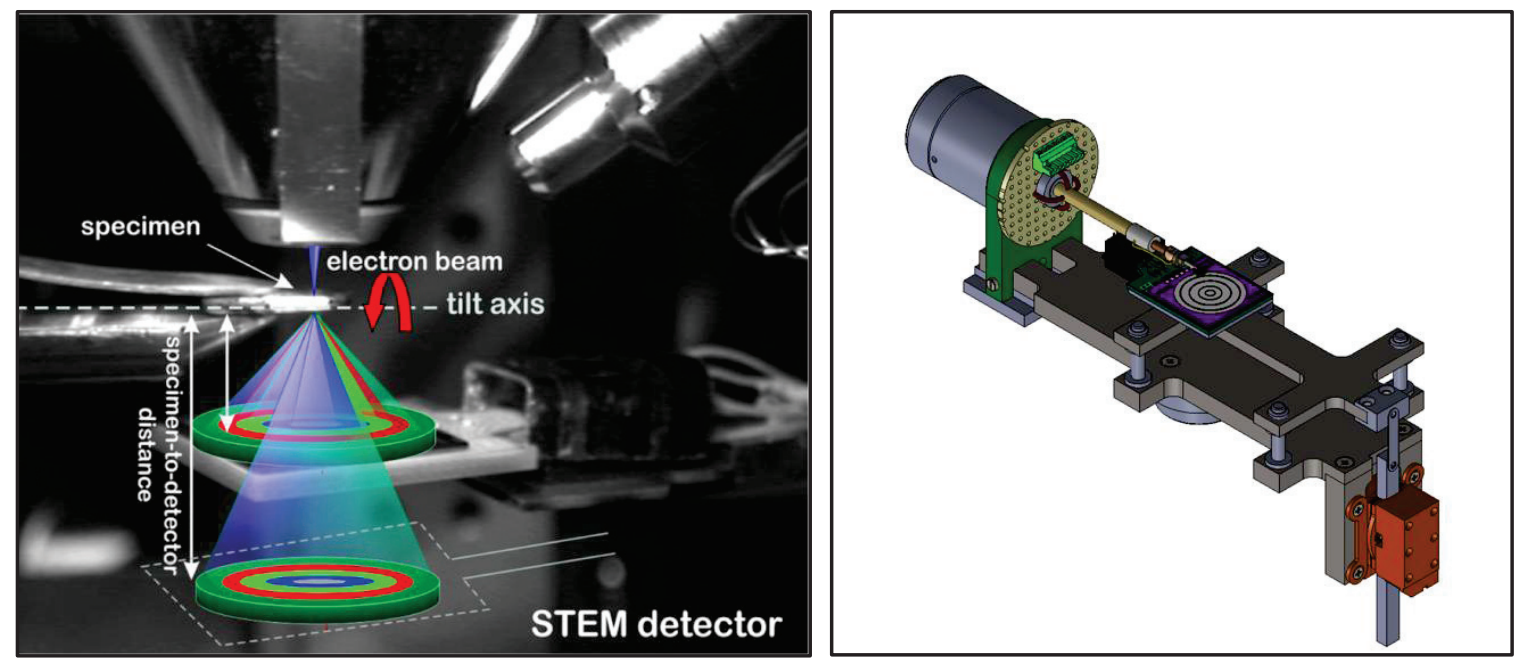

FIGURE 2 (Left) Setup inside the SEM-chamber: The specimen in mounted on a rotation holder and the STEM detector with adaptable working distance. The two colour sketches show the range for positioning the STEM detector. The specimen can be rotated along a horizontal tilt axis. (Right). Layout of the specimen rotation holder with eucentric capability. Complete rotation with eucentric adjustment and motorized specimen-detector distance are implemented.

Figure 2 shows the prototype of the tomographic acquisition system. On the left, the STEM detector is presented. The detector geometry with five independent circular active sectors permits to maximize the efficiency of signal collection. The colour sketch in the figure shows the adjustable geometry for the detector and highlights the outer and inner detection solid angles for the scattering cone of transmitted electrons. The active sectors of the detector allow separating between the Bright- and Dark-Field components of the signal, a basic feature to optimize the visibility and signal-to-background ratio for the specimen details. Moreover, the dedicated analogue/digital signal processing system was designed to guarantee amplification, conditioning and managing of the signals. Therefore, the bright-field component of the transmitted electrons can be effectively separated from the dark-field one, allowing one to adapt the detection strategy to the specimen characteristics $[7,8]$.

\section{Specimen rotation holder}

The dedicated specimen holder with rotation capability ensures the eucentricity of the observed detail and a reduced missing wedge, which are important for a practical implementation of the tomographic technique. The layout of the holder is shown in the right part of figure 2 .

The holder consists of a horizontal rod mounting a piezo-actuated spherical joint in proximity of the termination. The rod can be rotated with 0.1 degree precision, while the spherical joint can be adapted to drive the region of interest in correspondence of the rotation axis of the holder. When the sample remains at the intersection between the optical axis of the imaging system and the rotation axis of the specimen rod - a geometrical condition defined as the eucentric position - the region of interest is maintained in the field of view of the microscope upon tilting the sample.

The holder is fabricated to mount thin samples, either self-supporting specimens with thinned central part or grid supported powdered specimens. The $3 \mathrm{~mm}$ maximum diameter was considered, similarly to the TEM standard specimens. The mechanical mounting of the specimen has been carefully designed in order to contain the limitation in maximum tilting angle, above which a missing wedge for the directions of projection is introduced. 


\section{RESULTS AND DISCUSSION}

Finally, the method for the $3 \mathrm{D}$ reconstruction of micro and nano-structures is presented. A series of projections is acquired by tilting the sample through $-60^{\circ}$ to $+60^{\circ}$ from the horizontal position at $1^{\circ}$ step. The best image contrast is obtained for the Dark-Field imaging condition, where the central/undeflected part of the transmitted beam is not collected. The annular active area of the STEM detector allowed visualizing the sample as a bright signal over a dark background.

In Figure 3 (Upper-Left), a STEM image from the tilt series of a Quantifoil ${ }^{\circledR}$ TEM support membrane is presented. The membrane features regularly shaped circular apertures. The STEM image, being acquired at $55^{\circ}$ tilt from horizontal position, clearly shows a bright contrast along half of the edge of the apertures, the other half presenting a less perceptible contrast at the edge. A marked bright contamination spot is also visible at the centre of the image.
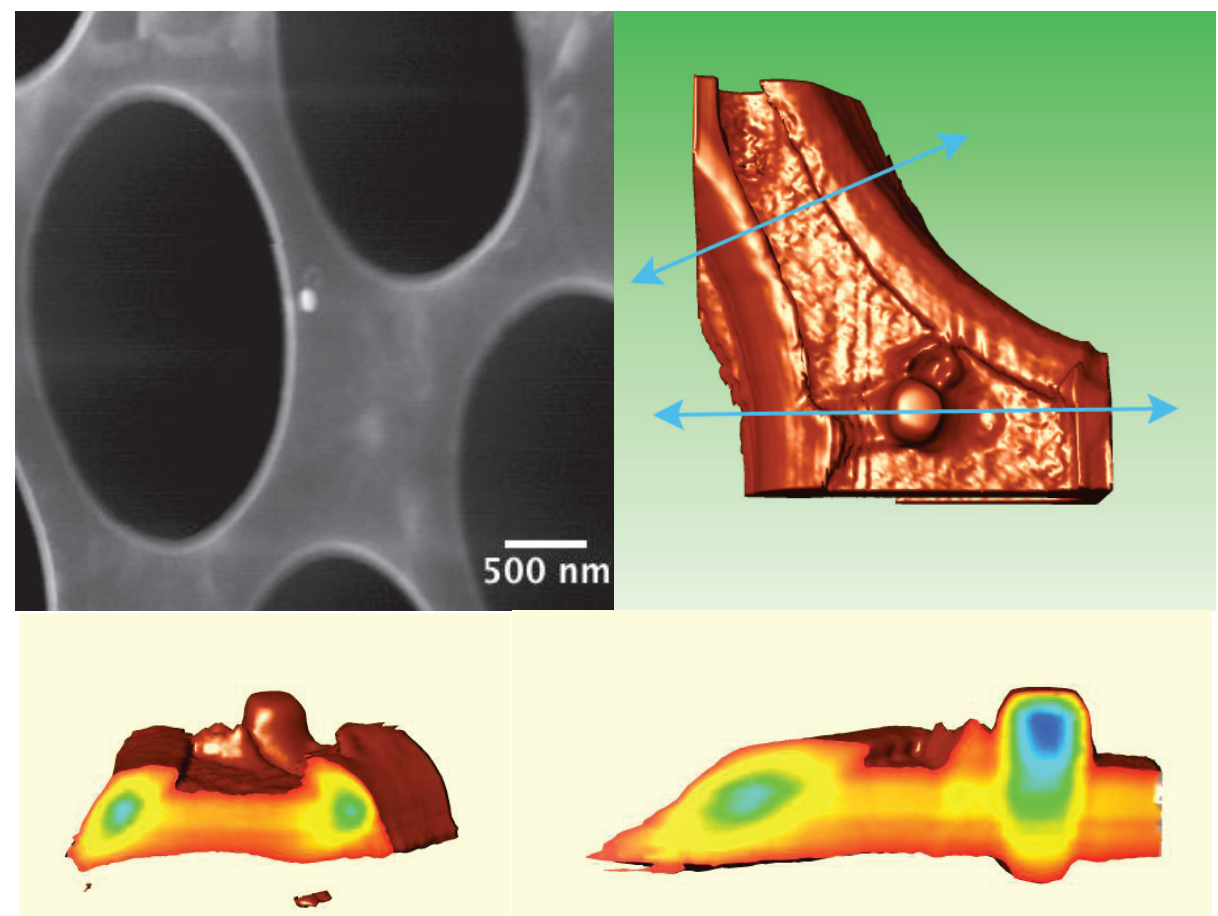

FIGURE 3. (Upper-Left) Steps for the tomographic reconstruction of a thin specimen. (a) single STEM image of a TEM support membrane. (Upper-Right) Top-view of the surface rendering of the reconstructed tomogram. (Bottom) Two vertical slices along the blue lines across the region of interest colored according to the voxel mass-density.

As the contrast in the STEM Dark-Field image depends on the projected thickness, the contrast modulation at the edge signifies an out-of-plane shape for the membrane. After a preliminary alignment of the experimental tiltseries, obtained from the set-up, the reconstruction was retrieved by ImageJ with the TomoJ plug-in [10, 11].

Figure 3 (Upper-Right) renders the tomographic reconstruction for the central region of interest. The top-view visualization of the tomogram is clearly similar to the STEM image, with evidence of interesting modification of the sample at the edges. The two slices of the tomogram presented in the lower part of the figure reveal the actual shape and thickness profile for the membrane, together with the shape of the contamination spot, differently dense with respect to the membrane.

Figure 4 shows a number of STEM Dark-Field projections at different tilt angle for a polymer fiber suspended on the carbon membrane [12]. The visible region measures $6 \mu \mathrm{m} \times 3.5 \mu \mathrm{m}$ in size. The corresponding tomogram, obtained by iterative tomographic reconstruction (SIRT) clearly shows the hollow cylindrical shape of the fiber. 

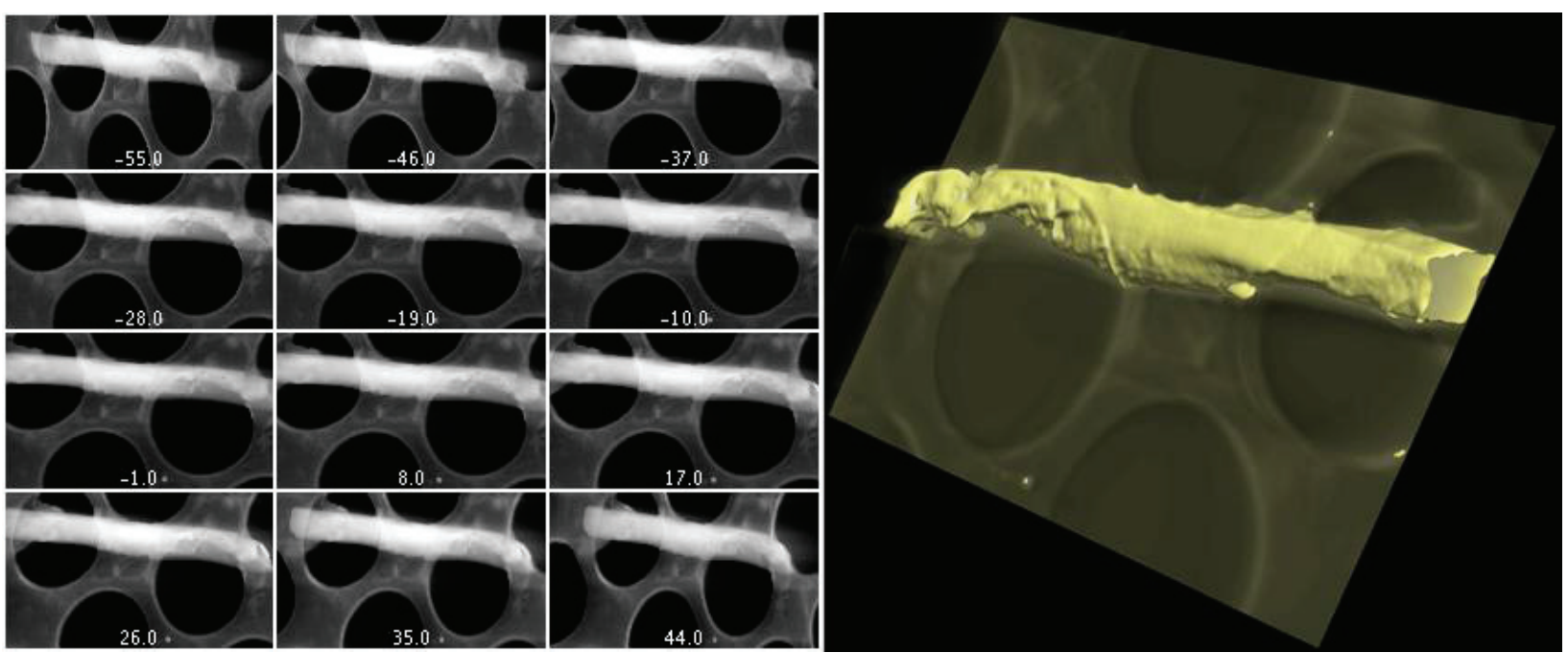

Figure 4: (Left) Projections from the tilt series polymer fiber. (Right) 3D-surface rendering of the fiber.

\section{CONCLUSIONS}

The proposed approach to SEM tomography demonstrates the perspective for the 3D analysis of volumes down to $100 \mu \mathrm{m}^{3}$, depending on specimen density and composition. According to Crowther's criterion, the tomogram resolution is determined by the number of available projections, which are assumed to be noise-free, perfectlyaligned, and covering the entire angular range [13]. In the practical case, the spectral signal-to-noise ratio, the residual projection misalignment, and the missing wedge decrease resolution, and the limit in resolution is posed by the probe size of the microscope and the number of projections that can be acquired without significant beam damage of the sample. For the sake of a quantitative estimation, a resolution of about $10 \mathrm{~nm}$ can be favorably estimated for the proposed technique. The proposed system performs at the intermediate volume and resolution range compared to the standard techniques, and exploits the flexibility of the SEM platform and the capability, in terms of resolution and image quality, of the scanning transmission imaging mode.

The proposed method is not destructive, leaving the sample in its pristine condition, and it can be potentially applied for both biological and materials science fields, first of all for nanowires or carbon-based nanostructures. It is worth noting that the experimental set-up is under further development to consolidate this technique in the ensemble of 3D methods of electron microscopy.

\section{ACKNOWLEDGMENTS}

This work was carried out within the TomoSEM Project (Identification number: F97I12000120007); the financial support from the FILAS Research Program is gratefully acknowledged.

\section{REFERENCES}

1. P.A. Midgley, M. Weyland, T.J. Yates,I. Arslan, R.E. Dunin-Borkowski, J.M Thomas, Journal of Microscopy 223, 185 (2006)

2. S. Bals, G. Van Tendeloo, C. Kisielowski, Advanced Materials 18, 892 (2006)

3. M.D. Uchic, L. Holzer, B.J. Inkson, E.L. Principe, P. Munroe, MRS Bullettin 32, 408 (2007)

4. A.C. Kak and M. Slaney in Principles of Computerized Tomographic Imaging, IEEE Press (1998), pp. 4956. 
5. J. Mahamid and W. Baumeister, Microscopy and Analysis, September Issue (2012) pp.45-48.

6. G. Mobus and B.J. Inkson, Materials Today 10, 18 (2007).

7. P.G. Merli, A. Migliori, V. Morandi, R. Rosa, Ultramicroscopy 88, 139 (2001).

8. V. Morandi, P.G. Merli, D. Quaglino, Applied Physics Letters 90, 163113 (2007).

9. V. Morandi and P.G. Merli, Journal of Applied Physics 101, 114917 (2007).

10. http://rsbweb.nih.gov/ij/ - ImageJ: Image Processing and Analysis in Java

11. C. Messaoudii, T. Boudier, C.O. Sanchez Sorzano, S. Marco, BMC Bioinformatics 8, 288 (2007).

12. N. Crivillers, L. Favaretto, A. Zanelli, I. Manet, M. Treier, V. Morandi, M. Gazzano, P. Samorì, M. Melucci, ChemmComm 48, 12162 (2012).

13. J. Frank, Electron Tomography. Springer Science \& Business Media, 2008. 